\title{
Nano-Kinetics of Probe-Particles in Solution Visualized by a Pin-Fiber Video Scope
}

\author{
Masashi Suzuto, * Yasuyuki Hirakawa,* Hitoshi Ohnishi,* Shinji Tachino,* \\ Tomoteru ShINGaKI,* Edward M. Eyring,** and Tsutomu MaSUJIMA **ं \\ *Graduate School of Medical Science, Hiroshima University, 1-2-3 Kasumi, Minami-ku, \\ Hiroshima 734-8551, Japan \\ **Department of Chemistry, University of Utah, Salt Lake City, UT 84112, USA
}

\begin{abstract}
The nano-kinetics of colloidal particles and living cells with the colloidal particles were visualized by a newly developed video scope. The system of the new video scope has a feature of fine controlling the illumination conditions by using a single optical fiber. This characteristic enables one to obtain clear images of living cells and the motions of colloidal particles by light-scattering effects. In the experiments, RBL-2H3 cells and gold colloidal particles were observed. Scattering images with high contrast and a dark background like in dark-field observations could be attained. In the experiments, a pulsed laser was also applied. The results obtained in this study could validate the effectiveness and possibility of a new video scope for applications to biological and biomedical fields.
\end{abstract}

(Received October 7, 2002; Accepted November 21, 2002)

\section{Introduction}

Single molecule imaging of biological molecules is having a strong impact on the fields of biology and biomedicine. Recently, the scheme of single-molecule imaging has been applied to living cells, and a great number of remarkable results have so far been reported. ${ }^{1-5}$ These single-molecule imaging studies were performed based on a near-field illumination technique. Near-field illumination utilizes an evanescent field generated by total reflection at an interface, or by a microscopic optical aperture. The area where an evanescent field can be used is limited to around an interface between two different optical media. This indicates that the region that we can observe with an evanescent microscope is restricted to some extent. In order to overcome this problem, some fully workedout schemes have been proposed. ${ }^{4}$

An optical fiber is very flexible and has a high transmission of visible and infrared light. When the exit of an optical fiber is set in the vicinity of a sample of an optical microscope, a clear and bright illumination of the sample is possible. This method needs no interface to generate an evanescent field, because it is not based on a near-field observation. The method using optical fibers can utilize a coherent/incoherent light source for normal optical microscopes.

In this paper, a new video-microscope using a single optical fiber as an illumination source is presented. We named this system "pin-fiber video scope (PFVS)". A prototype PFVS was fabricated and tested by observations of gold colloidal particles (g-particles) and living cells with a light-scattering effect. From this basic study, it was found that PFVS can be a powerful tool for single molecule imaging, and other biological/biomedical observation.

$\dagger$ To whom correspondence should be addressed.

E-mail: tsutomu@hiroshima-u.ac.jp

\section{Experimental}

The experimental setup for this study is shown in Fig. 1. The main experimental apparatus was the PFVS. The samples observed were g-particles and rat basophilip leukemia (RBL$2 \mathrm{H} 3$ ) cells. Images obtained in this study were caused by lightscattering phenomena. They are known as Rayleigh scattering for g-particles, and Mie scattering or geometrical optics scattering for living cells.

\section{Pin-fiber video scope (PFVS)}

The PFVS system consists of three major parts. They are namely a light source, a single optical fiber, and a fundamental optical microscope. The light used for illuminating samples in the microscope is supplied throughout the optical fiber. Although a laser source is the best light source, since a better coupling efficiency between the light and the fiber is easily attained, a conventional white light source, such as a halogen lamp and a mercury lamp, is also available. White light filtered by a band-pass filter (excitation filter) can also be used. The

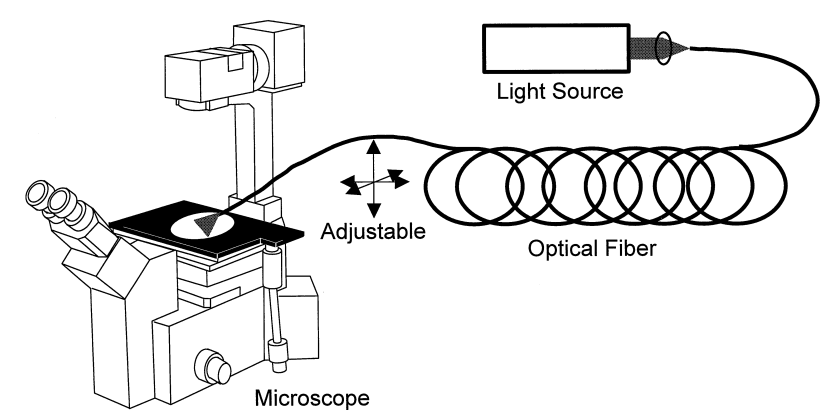

Fig. 1 Experimental setup of a pin-fiber video scope (PFVS). 


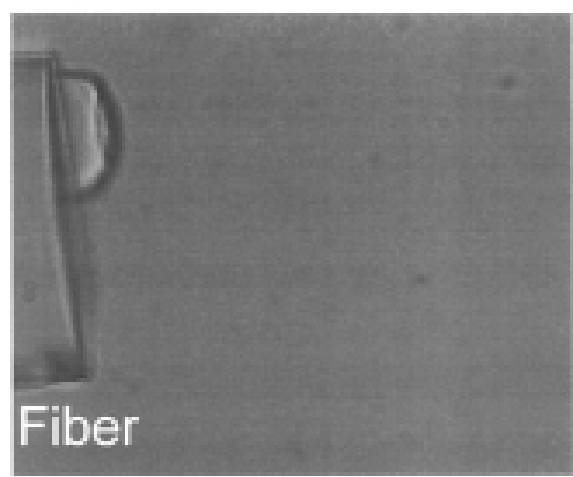

(a)

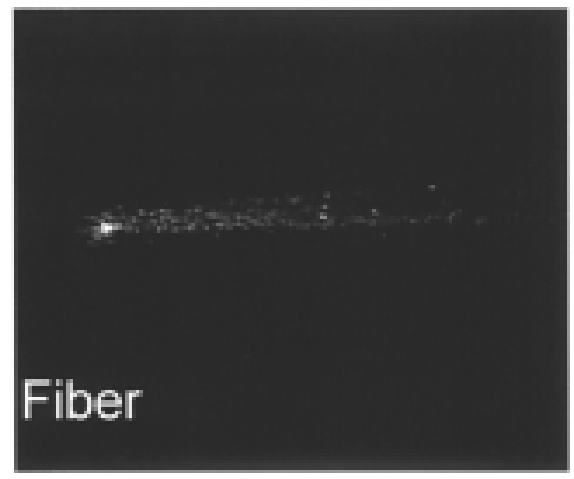

(b)

Fig. 2 Observation of g-particles; (a) with a normal bright vision, (b) with PFVS.

PFVS has several advantages as follows.

Controllability of the illumination area. The illuminated area of the sample can be controlled by moving the fiber. Controlling the distance between the fiber exit and the sample permits a change in the illuminated area and the illumination intensity. A limited illuminated area might be useful when a single molecule in a living cell is observed with this system, because unnecessarily bleaching of fluorescent molecules illuminated by a normal fluorescence microscope system can be avoided. As a result, the observation of the cell can be continued by changing the observed molecule in the cell.

Two imaging modes. A scattering image and a fluorescent image are available. Although a normal fluorescent microscope offers only fluorescent images, with PFVS, scattering images that are not identical to images obtained by a bright view observation (explained in a following part) can be obtained in addition to fluorescent images. This is due to focusing the light onto only the sample by the optical fiber. As a result, weaker images under a bright view observation with normal optical microscopy can be enhanced and are easily observed. It is, of course, applicable to fluorescent observations, and it is possible to obtain enhanced fluorescent images by PFVS.

No scanning procedure. A scanning procedure, which is essential when using a laser confocal microscope and a scanning microscope, is not necessary. The illuminated spot size on the sample is variable by changing the distance between the sample and the exit of the fiber, as described above. When using a wide area of illumination, as when a normal bright field observation is needed, one only has to enlarge the distance between the fiber and the sample. When a very small illuminated area is preferable, a spot diameter of $\sim 1 \mu \mathrm{m}$ on the sample is feasible by approaching the fiber exit to the sample as closely as possible.

Easy installation. It is easily possible to attach this system to existing optical microscopes in laboratories. All additional apparatuses are externally attached, and it is not necessary to make modifications of the microscope optics.

Inexpensive. It is essentially inexpensive. The only things needed, except for the light source, are a single fiber, its coupling optics, and a micro-manipulator. The fundamental microscope does not need to be an expensive laser confocal microscope or a scanning microscope. Normal upright or inverted microscopes are available as the fundamental microscope.

\section{Instrumentation}

In the present experiment, a He-Ne laser (MellesGriot, 05LGR-193, $543.5 \mathrm{~nm}, 5 \mathrm{~mW}$ ) was used as the light source. The coupling optics to focus the laser beam into the optical fiber (Thorlabs, SM500) entrance was an aspherical lens for fiber optics (Thorlabs, A390TM-A), which attained a coupling efficiency of $30 \%$. A Nd:YAG laser (HOYA-Continuum, Minilite II, $532 \mathrm{~nm}, 25 \mathrm{~mJ}, 10 \mathrm{~Hz}, 5 \mathrm{~ns}$ ) was used instead of a continuous laser when the pulsed-light effects from the fiber were estimated. As for the fiber, a multi-mode fiber (Thorlabs, AFS105/125Y) was utilized in the case of pulsed laser irradiation. The laser power and the illumination power with an optical fiber were measured using a laser power meter (Molectron, PM3, MAX500A). The illuminated area with light from the optical fiber was adjusted by a micro-manipulator (Narishige, NHN-21 and MHW-4). The microscope used in the present experiments was the upright system type (Zeiss, Axioplan). The generated image of the sample was detected by a video camera (SONY, SSC-DC430). The detected image was recorded by a digital video recorder (SONY, DSR-30), and finally the image was processed with computer software (Adobe, Photoshop; National Institute of Health, NIH images; and Apple, iMovie) to enhance the image for image analyses.

\section{Material and reagents}

G-particles were obtained by the reduction chloroauric acid by sodium citrate. $^{7}$ Then, $0.50 \mathrm{~mL}$ of $1 \%$ sodium citrate (Katayama) was added to a boiled $50 \mathrm{~mL}$ portion of $0.01 \%$ chloroauric acid (Nacalai Tesque). The solution was kept boiling until it showed a wine-red color. The g-particles obtained were pipetted on a glass slide (Mastunami Glass, 76 $\mathrm{mm} \times 26 \mathrm{~mm}, 1.0 \mathrm{~mm}$ thick), which was cleaned with distilled water in advance. RBL-2H3 cells were incubated by a method described in a reference, ${ }^{7}$ and DNP-BSA (Sigma, $20 \mathrm{ng} / \mathrm{mL}$ ) was used as an antibody.

\section{Results and Discussion}

\section{G-particles observation}

A scattering image of g-particles was observed in order to confirm the validity of this novel video scope. An image obtained under a normal bright-field is shown in Fig. 2 (a), in which an edge of the optical fiber can be seen on the left-hand side. In front of the fiber edge, which was the exit of illumination light, numerous g-particles were expected to be 


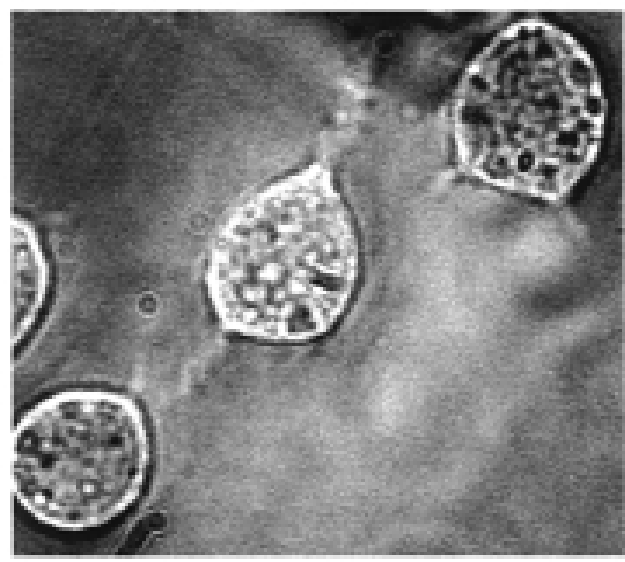

(a)

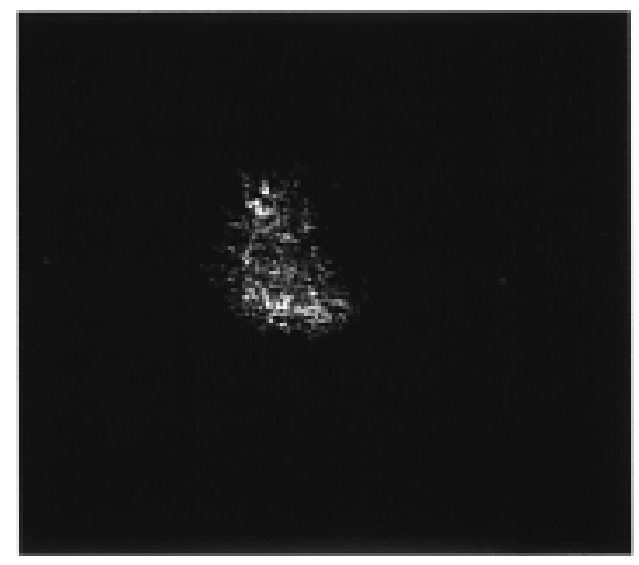

(b)

Fig. 3 Application of PFVS to living cells; (a) with normal bright vision, (b) with PFVS. A high contrast and a dark background image could be obtained by PFVS.
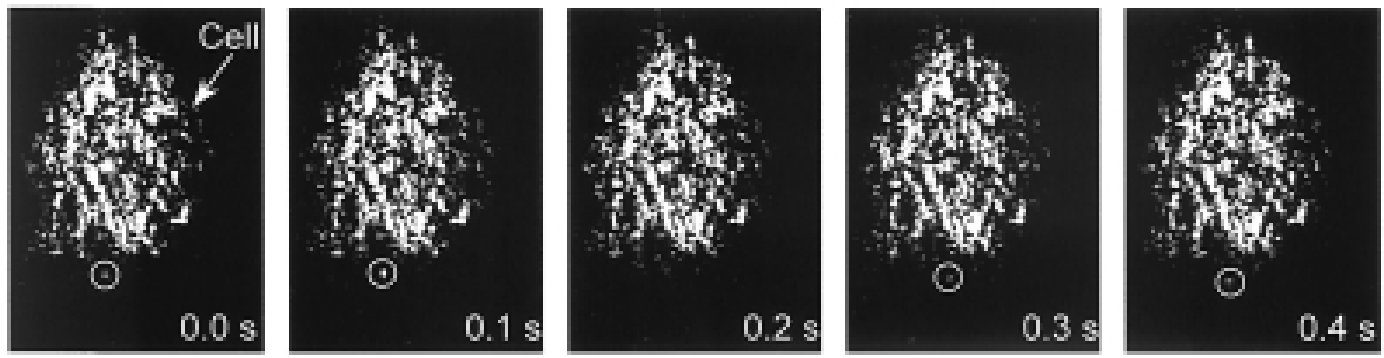

Fig. 4 Motion of an antibody modified by a g-particle visualized by PFVS around a cell. The white spot surrounded by the open circle is a g-particle. The time codes are also indicated in each picture.

moving around with Brownian motion. However, almost no particle was clearly visible in this experiment. When the laser was turned on and the laser light came out from the exit of the fiber, the g-particles were illuminated and the light was scattered by their surfaces. Brownian movement of the $\mathrm{g}$ particles was clearly observed, and each g-particle was clearly recognized as a bright light point, as shown in Fig. 2 (b). This scattering phenomenon should be Rayleigh scattering considering the size of the particles and the wavelength of the laser light in this case.

\section{Living cells observations}

The imaging of living cells by PFVS was conducted. The cells used were RBL-2H3, whose size was comparable to the core diameter of the optical fiber. Figure 3 (a) shows a normal bright-field image irradiated with a halogen lamp. When the halogen lamp was turned off, and one of the cells in the same view field was illuminated by the fiber, a clear image of the cell appeared as shown in Fig. 3 (b). It is obvious that the background of the image was completely dark, just as in a darkfield microscopic observation. This is one of the PFVS advantages, that images with a high signal-to-noise ratio can be produced, especially in the case of a scattering observation, as in Fig. 3 (b).

Movements of RBL-2H3 cells stimulated by antibodies modified with g-particles were observed by PFVS. Although the antibody, DNP-BSA, could not be seen directly by PFVS, gparticles were visualized with a scattering-light. It was

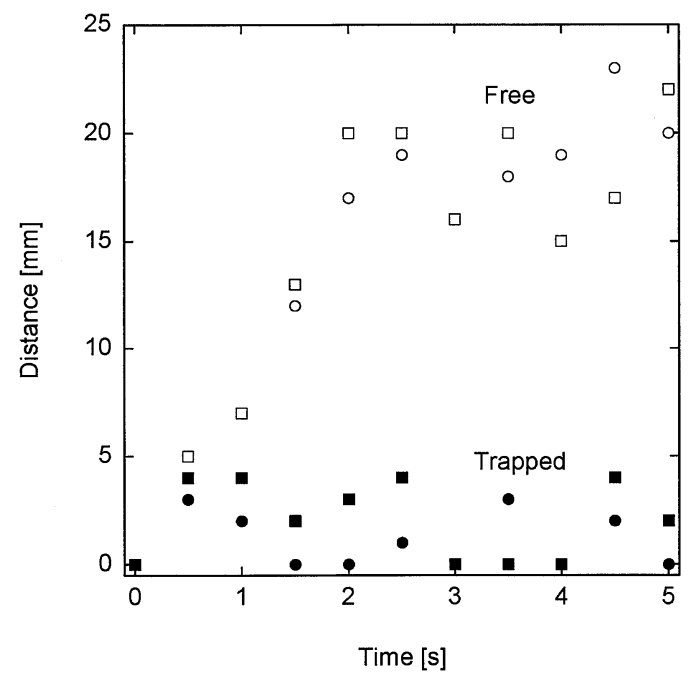

Fig. 5 Difference in the total moving lengths between g-particles with different distances from the cell.

expected that the image of an interaction between the antibody and the cell is possible by PFVS. Figure 4 illustrates the results of the observation together with their time codes. One gparticle attached to one DNP-BSA molecule, which is indicated by an open circle in the figure, was moving in the vicinity of the 

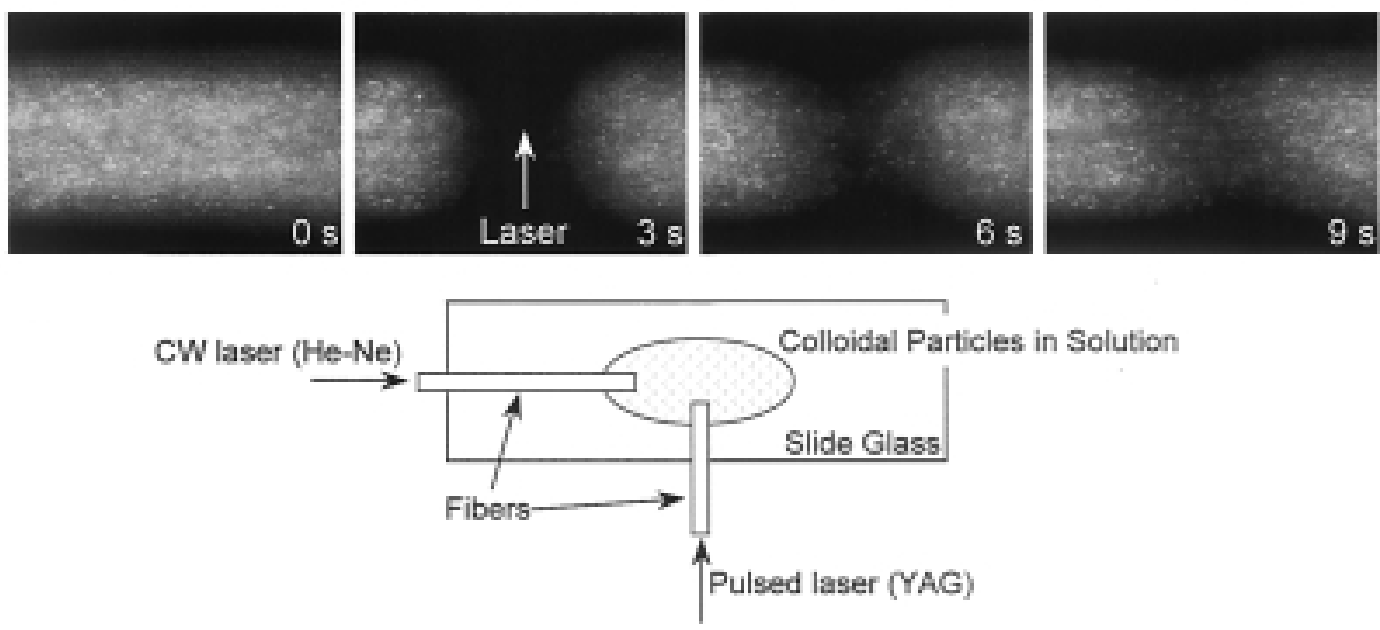

Fig. 6 Effect of irradiation by pulsed laser light to g-particles. The arrow indicates the laser path of the pulsed laser, and the time codes are indicated in each picture. A schematic drawing of the setting is also shown.

cell. When $t=0.2 \mathrm{~s}$, the particle disappeared suddenly, and at $t$ $=0.3 \mathrm{~s}$ it appeared again in the visual field. The movement of this colloidal particle was not as active as other particles moving far from the cell. Figure 5 shows the difference in the total moving lengths of g-particles with different distances from the cell. The moving length of the g-particles in the vicinity of the cell is extremely shorter than that of the particles far from the cell. This means that particles near to the cell might be trapped with a receptor on the cell, while particles far from the cell were free to move around with Brownian motion. This visualization experiment suggests that the PFVS system could be a superior tool for biological and biomedical applications in which microscopic observations of living cells are essential.

\section{Application of a pulsed laser as a light source}

The normal laser light source for PFVS is a continuous-wave (CW) laser. It is suitable to couple with an optical fiber without any damage caused by powerful laser irradiation. However, for applications that need a peak power or a high temporal resolution, it is possible to utilize a pulsed laser in the PFVS system instead of CW lasers. In this study, a pulsed Nd:YAG laser was used as a light source, and its output was injected into a muti-mode fiber with an energy of $1 \mathrm{~mJ} /$ pulse and a duration of $5 \mathrm{~ns}$. The axis of this fiber was set to be perpendicular to another fiber for the CW laser, as shown in Fig. 6. The light from the fiber for the $\mathrm{CW}$ laser irradiated the g-particles and produced a scattering image, while the other fiber for the pulsed YAG laser illuminated the same g-particles in the perpendicular direction. When the particles were irradiated by light from the pulsed laser, the particles suddenly disappeared from the visual field. After a few seconds, the dark part, in which the gparticles disappeared, was soon covered with other g-particles by particle diffusion. This phenomenon means that the gparticles received some optical power, and that they were moved away or completely destroyed. Since the absorbance of the g-particles is 0.8 at $543 \mathrm{~nm},{ }^{8}$ it is reasonable to think that the g-particles absorbed the optical power and broke into small pieces. Although the process of the breakdown is not clear, photothermal melting 9 or a shock-wave effect ${ }^{10}$ is possible. The pulsed energy of the laser was $1 \mathrm{~mJ}$, which corresponded to 2.5 $\mathrm{MW} / \mathrm{cm}^{2}$ at the focus of the illumination light, might cause an explosion of the g-particles. This phenomenon is interesting from the point of view of medical applications, because the g- particle, whose diameters are usually larger than the attached molecules investigated and their size effects are not negligible, can be made to disappear by only irradiating several laser pulses when g-particles are no longer necessary. Unnecessary effects by g-particles could be suppressed by this method.

\section{Optimization of scattering images}

The scattering phenomenon in the case of g-particles was Rayleigh scattering considering the particle size. The intensity of the Rayleigh scattering $I_{\text {Rayleigh }}$ for an incident intensity of $I_{0}$ is given by $I_{\text {Rayleigh }} \propto I_{0} / \lambda^{4} r^{2}$, where $\lambda$ and $r$ are the wavelength of the light and radius of the particles, respectively. ${ }^{11}$ In order to increase the scattering signal, it is better to use intense illumination with light of a shorter wavelength, as this equation predicts. The scattering by a living cell is Mie scattering or geometric optical scattering. In this case, the form of the sample (cell) is a critical factor, and the irradiating power is also important as in the Rayleigh-scattering case. However, for both cases, an excessive strong illumination power probably gave some damages to the samples, and light with shorter wavelengths, such as ultraviolet radiation, is harmful for living samples. It should be noted that the optimum intensity and wavelength of the illumination light must be selected carefully.

Another method to obtain better imaging is the application of a fast Fourier transformation (FFT) to the image. By subtracting lower frequency components from the image after FFT, a clear image without small noises could be attained by reversing into the original Fourier domain. This technique is useful for an image with white noise or a small amount of scattering noise.

\section{Conclusion}

We demonstrated visualizations of g-particles and living cells by a novel video-microscope, a pin-fiber video scope, using a single optical fiber. The main merit of the PFVS is to precisely control of the illumination condition, including the area and intensity. The Brownian motion of g-particles, which is almost impossible to observe with a normal bright field, could be clearly visualized. In the case of cell observations, the cell images were observed with a high signal-to-noise ratio just as in a dark-field microscopic observation. With additional 
antibodies modified by g-particles, it was found that microscopic observations of the interaction between the cell and the anti-body using a scattering image are feasible. These obtained results suggest that the PFVS system has a potential for being a superior analytical tool for both biological/biomedical investigations and applications.

\section{Acknowledgements}

This research was partially supported by the Japan Ministry of Education, Science, Sports and Culture, Grant-in-Aid for Scientific Research

\section{References}

1. S. Weiss, Science, 1999, 283, 1676.

2. X. Xu and E. S. Yeung, Science, 1997, 276, 1106; "Trends in Analytical Life Sciences", 1997, 1, 173 (http://neo.pharm.hiroshima-u.ac.jp/tals/).

3. K. Kitamura, M. Tokunaga, A. H. Iwane, and T. Yanagida, Nature, 1999, 397, 129.

4. H. Tadakuma, J. Yamaguchi, Y. Ishihama, and T. Funatsu, Biochem. Biophys. Res. Commun., 2001, 287, 323.

5. T. Fujiwara, K. Ritchie, H. Murakoshi, K. Jacobson, and A. Kusumi, J. Chem. Biol., 2002, 157, 1071.

6. G. Frens, Nature (Physical Science), 1973, 241, 20

7. K. Ozawa, H. Kobayashi, E. Kawai, E. Suzaki, Y. Nonomura, and T. Masujima, FEBS Lett., 1996, 398, 67.

8. C. A. Foss, Jr., G. L. Hornyak, J. A. Stockert, and C. R. Martin, J. Phys. Chem., 1994, 98, 2963.

9. S. Link, C. Burda, M. B. Mohamed, B. Nikoobakht, and M. A. El-Sayed, J. Phys. Chem. A, 1999, 103, 1165.

10. M. Nakahara and K. Nagayama, Technology Reports of Kyushu University (in Japanese), 2001, 74, 653.

11. R. M. Measures, "Laser Remote Sencing", 1984, John Wiley \& Sons, New York, 46. 